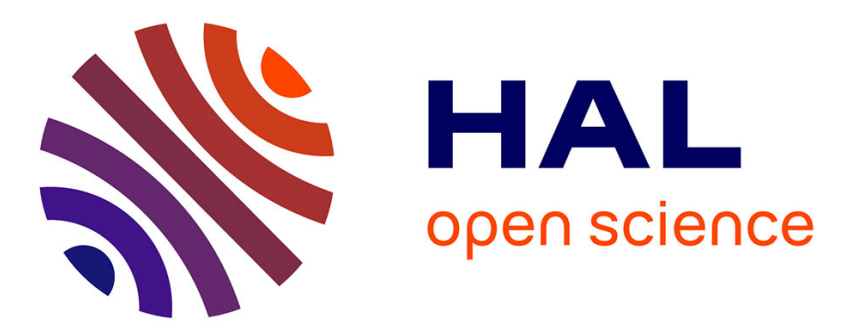

\title{
Quelles références pour enseigner la transition agroécologique ? Étude de discours de chercheurs sur leurs pratiques scientifiques
}

\author{
Nicolas Hervé, Nathalie Panissal, Nadia Cancian
}

\section{To cite this version:}

Nicolas Hervé, Nathalie Panissal, Nadia Cancian. Quelles références pour enseigner la transition agroécologique ? Étude de discours de chercheurs sur leurs pratiques scientifiques. Éducation \& Didactique, 2021, 15 (15-2), pp.103-123. 10.4000/educationdidactique.8769 . hal-03299033

\section{HAL Id: hal-03299033 https://hal.science/hal-03299033}

Submitted on 25 Jul 2021

HAL is a multi-disciplinary open access archive for the deposit and dissemination of scientific research documents, whether they are published or not. The documents may come from teaching and research institutions in France or abroad, or from public or private research centers.
L'archive ouverte pluridisciplinaire HAL, est destinée au dépôt et à la diffusion de documents scientifiques de niveau recherche, publiés ou non, émanant des établissements d'enseignement et de recherche français ou étrangers, des laboratoires publics ou privés. 


\title{
Quelles références pour enseigner la transition agroécologique ? Étude de discours de chercheurs sur leurs pratiques scientifiques
}

\author{
Nicolas HERVÉ \\ Ecole Nationale Supérieure de Formation de l'Enseignement Agricole, UMR EFTS, \\ Université de Toulouse \\ nicolas.herve@ensfea.fr \\ Nathalie PANISSAL \\ Ecole Nationale Supérieure de Formation de l'Enseignement Agricole, UMR EFTS, \\ Université de Toulouse \\ nathalie.panissal@ensfea.fr \\ Nadia CANCIAN \\ Ecole Nationale Supérieure de Formation de l'Enseignement Agricole, UMR EFTS, \\ Université de Toulouse \\ nadia.cancian@ensfea.fr
}

\section{Résumé :}

Cet article explore la possibilité d'une transposition didactique des pratiques de recherche à propos de questions socialement vives liées à l'agroécologie, à partir de l'analyse d'entretiens de chercheurs. Les résultats montrent que les chercheurs mettent en avant quatre caractéristiques de la nature de la science en rapport avec l'agroécologie : l'interdisciplinarité entre les sciences humaines et sociales et les sciences agronomiques ; le caractère inductif et participatif de leur démarche scientifique ; l'alignement des valeurs sociales des chercheurs et de leur éthique professionnelle pour un engagement qui vise une transformation sociale ; la responsabilité épistémique vis-à-vis des grands enjeux environnementaux et sanitaires. Ces éléments nous permettent de proposer trois «balises curriculaires » pour l'enseignement de l'agroécologie.

Mots-clés : questions socialement vives, nature de la science, pratiques de chercheurs, agroécologie, transposition didactique

Title: What references to teach the agroecological transition? Study of researchers' discourse on their scientific practices.

Abstract:

This article explores the possibility of didactic transposition of research practices on socially acute questions linked with agroecology, based on analysis of interviews with scientists. The results show that the salient points of the nature of agroecology are the interdisciplinarity (human and social sciences with agronomic sciences), the inductive and participative nature of their scientific approach, the alignment of the social values of researchers and their scientific ethos, that deals with engagment for social transformation, the epistemic responsibility for major environmental and health issues. These elements allow us to propose three "curricular markers" for the teaching of agroecology.

Keywords: Socially acute questions, Nature of Science, Scientific practices, Agroecology, Didactic transposition

\section{Introduction}

Les savoirs et pratiques des scientifiques constituent un ensemble de références pour l'enseignement des sciences et des techniques, et bien que les travaux pionniers en didactique aient forgé des cadres analytiques pour en rendre compte (comme la transposition didactique 
ou bien les pratiques sociales de référence), la nature de ce lien reste toujours une question centrale et particulièrement ouverte.

Albe et Orange (2010) soulignaient déjà que le questionnement sur les références de l'enseignement scientifique et technique avait été renouvelé sous l'impulsion de plusieurs changements curriculaires :

- l'introduction de démarches pédagogiques prenant pour modèle l'activité des scientifiques (sous les termes de « démarche d'investigation » ou "démarche scientifique »),

- la mise à l'étude de savoirs épistémologiques (la « nature de la science »),

- l'émergence de questions socialement vives (QSV),

- ainsi que la multiplication des « éducations à ».

La question des références de l'enseignement scientifique et technique a sans doute gagné encore plus en complexité ces dernières années. Le paysage scientifique est marqué par le développement des technosciences (par exemple l'intelligence artificielle, les nanotechnologies ou les OGM). Les problématiques environnementales et sanitaires ont provoqué des reconfigurations disciplinaires sur de nouveaux objets (par exemple autour du système-terre ou de l'agroécologie). La prolifération de discours fallacieux singeant le fonctionnement des institutions scientifiques via les réseaux sociaux (comme le platisme ou le mouvement antivax), ou bien la mise au jour médiatisée de pratiques scientifiques malhonnêtes et contraires à l'éthique de la recherche (affaires des Monsanto Papers ou du Mediator) ont nourri cette complexité. Ainsi, de nouveaux cadres épistémologiques (Coutellec, 2015), sociologiques (Chateauraynaud, 2011), et anthropologiques (Vinck, 2007) se développent pour rendre intelligibles la production contemporaine des savoirs scientifiques et techniques et leur circulation dans l'espace social.

Nous estimons, avec d'autres chercheurs, que la didactique des sciences ne peut rester à l'écart de ces évolutions épistémiques. II s'agit alors d'actualiser et d'enrichir les arrière-plans épistémologiques qui permettent d'appréhender des changements dans la manière dont les sciences et techniques sont susceptibles d'être scolarisées.

El Hage et Ouvrier-Buffet (2018) défendent l'idée que ces évolutions rendent nécessaires un “ changement de posture épistémologique pour les chercheurs en didactique » (p. 109), et elles proposent de caractériser l'épistémologie des démarches de recherche menées par les scientifiques afin d'en extraire des références susceptibles notamment d'alimenter de nouvelles ingénieries didactiques. L'étude des pratiques de chercheurs peut ainsi permettre aux chercheurs en didactique d'assurer plus fortement leur "vigilance épistémologique » (Chevallard, 1985) à propos de l'enseignement de savoirs ou de pratiques aux contours flous et incertains, qui relèvent d'une « science en train de se faire » (Latour, 1989).

Cet article adhère à ce projet général en prenant le point de vue de la didactique des questions socialement vives. Nous problématisons dans un premier temps l'intérêt de construire les références de l'enseignement à partir des savoirs et pratiques de chercheurs, et présentons un cadre d'analyse épistémologique qui nous permet de rendre compte de pratiques de scientifiques à des fins de transposition didactique. En lien avec notre implication dans l'enseignement agricole, nous nous centrons ensuite sur le domaine de l'agroécologie, et décrivons la nature de la science que déclarent pratiquer des chercheurs d'une même unité. Nous envisageons finalement quelques éléments qui peuvent servir de «balises curriculaires » (Lange et Victor, 2006) pour l'enseignement de l'agroécologie en lycée agricole.

\section{Approches didactiques de caractérisation des pratiques contemporaines de chercheurs}

Peu de travaux s'intéressent aux références des savoirs et pratiques scientifiques scolaires en partant d'une description des activités des chercheurs. Les dispositifs institutionnels 
mettant en relation chercheurs et enseignants de façon à " recontextualiser » les savoirs et pratiques des chercheurs dans la classe sont également rares (Pommier, FoucaudScheunemann, et Morel-Deville, 2010).

Quelques travaux en didactique de la physique et des mathématiques ont tout de même étudié comment une modélisation à visée didactique de pratiques de chercheurs pouvait conduire à renouveler les références pour un enseignement scientifique (El Hage et Ouvrier-Buffet, 2018 ; El Hage et Plé, 2016 ; Gardes, 2013 ; Wong, Hodson, Kwan, et Yung, 2008 ; Wong, Kwan, Hodson, et Yung, 2009).

Ainsi, Gardes (2013) a modélisé les pratiques de recherche de deux mathématiciens à propos d'une conjecture en théorie des nombres à partir d'observations et d'entretiens, El Hage et Plé (2016) et El Hage et Ouvrier-Buffet (2018) ont caractérisé et comparé quelques éléments de pratiques de recherche de mathématiciens et de physiciens à partir d'entretiens semi-directifs. Wong et al. $(2008,2009)$ montrent comment un enseignement en formation d'enseignants portant sur la nature de la science a pu être conçu à partir d'entretiens de scientifiques ayant joué un rôle dans la prise en charge de l'épidémie du SRAS à Hong Kong en 2003. Dans ces différents cas, il s'agissait pour les chercheurs en didactique de construire des références sur les démarches de recherche de scientifiques, donc de "science en train de se faire ", afin d'en questionner les potentialités de transposition dans les pratiques d'enseignement, en particulier dans le cadre de situations de résolution de problèmes ouverts, de démarches d'investigation ou de questions socio-scientifiques. Dans cette perspective, l'intérêt pour les pratiques des chercheurs rejoint celui de la conception de situations d'enseignement dans lesquelles l'étude d'un problème ou d'une question est centrale. Ceci fait écho aux préoccupations didactiques portées par le cadre de la problématisation dans l'enseignement (Fabre, 2011). Dès lors, il apparaît intéressant de considérer la transposition des pratiques des chercheurs en étudiant les types de problèmes sur lesquels ils travaillent.

Bien entendu les pratiques scientifiques des chercheurs et celles en milieu scolaire sont nécessairement différentes, car elles prennent forme dans des institutions différentes (Calmettes, 2012), il s'agit donc de s'interroger sur le processus de transposition externe qui opère par l'identification et l'extraction de certaines pratiques de leur milieu d'origine, et leur potentielle reconfiguration pour s'adapter à l'institution scolaire. Les travaux précédemment cités se situent alors plus précisément dans le processus de décontextualisation de la transposition didactique externe (Astolfi et Develay, 1989), c'est-à-dire «le remplacement du référent scientifique original par un "espace théorique de substitution ", qui a toutes les caractéristiques imposées par le processus d'enseignement »(Paun, 2006, p. 5). En effet, comme le rappelle Joshua (1996), « la transposition ne peut prendre directement des pratiques comme référence, du moins non sans un tamis de type théorique " (p. 65), c'est pourquoi les travaux se sont attachés à mobiliser ou à construire des outils conceptuels qui jouent le rôle d'une théorie " intermédiaire » permettant une modélisation des pratiques des chercheurs à des fins didactiques. Une théorie "intermédiaire » (au sens de Tiberghien, 2011 ; Veillard, Tiberghien, \& Vince, 2011) a en effet pour caractéristiques de se fonder à la fois sur des théories générales (relatives aux savoirs et à leur épistémologie, à leur enseignement et leur apprentissage), ce qui la rend consistante avec ce qu'elle cherche à décrire, et également de constituer un cadre théorique adapté au cadre scolaire, notamment car elle permet d'orienter et de baliser la conception de programmes d'enseignement ou d'activités pédagogiques.

C'est en tant que théorie intermédiaire que Gardes (2013) conceptualise des "gestes didactiques » pour rendre compte du processus de recherche de deux mathématiciens, que El Hage et Ouvrier-Buffet (2018) mobilisent le modèle cKф (conception, connaissance, concept) de Balacheff (2003) pour comparer ce que disent des scientifiques de leurs pratiques de recherche. De même, Wong et al. (2008) se servent des dimensions de la nature de la science décrite par McComas, Clough, et Almazroa (1998) pour catégoriser les entretiens des chercheurs. 
Deux perspectives épistémologiques peuvent toutefois être prises dans le choix de la théorie intermédiaire à mobiliser. Elle peut être plutôt de type " internaliste ", c'est-à-dire qu'elle rend compte des pratiques de scientifiques en décrivant les dynamiques de raisonnement, les débats d'idées, les justifications construites (on peut ranger dans cette catégorie les travaux d'El Hage et Ouvrier-Buffet, 2018 ; El Hage et Plé, 2016 ; Gardes, 2013). L'autre perspective, plutôt de forme "externaliste ", inscrit l'activité scientifique dans le fonctionnement de ses institutions et des contextes sociaux (comme le font Wong et al., 2008). Ces deux possibilités épistémologiques renvoient en fait aux dimensions de la nature de la science que les chercheurs en didactique souhaitent mettre en évidence dans les pratiques des scientifiques. Pour éclairer le choix à faire dans une théorie intermédiaire permettant de rendre compte des pratiques de recherche sur une QSV, il nous est nécessaire ici de nous situer dans les débats actuels des recherches concernant la nature de la science (NOS - Nature of Science).

\section{Des controverses sur la NOS marquant un intérêt croissant pour les pratiques contemporaines des chercheurs}

De nombreuses recherches mettent aujourd'hui l'accent sur le besoin de former élèves et étudiants à des pratiques scientifiques qui sont en lien avec les problématiques sociétales (Tytler, 2007 ; Hodson, 2011 ; Simonneaux, 2017).

Dans ce contexte, plusieurs courants d'éducation aux sciences convergent pour promouvoir un enseignement des sciences et des techniques qui vise à développer chez les apprenants des "visions plus holistiques et critiques des relations entre le développement des sciences et des technologies et de nombreux problèmes environnementaux, personnels et sociaux » afin de penser le monde de façon plus juste et soutenable (Bencze et al., 2020, p. 2). II s'agit du courant STSE, qui étudie les relations entre les sciences, la technologie, la société et l'environnement, de celui des questions socio-scientifiques (SSI - socioscientific issues) et de la didactique des questions socialement vives (QSV).

L'approche éducative de la nature de la science (NOS) - telle qu'elle est formalisée depuis les années 2000 à partir des travaux fondateurs de McComas, Clough, et Almazroa (1998), Lederman (2007) et Abd-El-Khalick (2012) - est critiquée parce qu'elle ne rend que partiellement compte de la réalité de l'activité scientifique (Hodson et Wong, 2017), et plusieurs chercheurs en éducation appellent à son actualisation.

Les principales caractéristiques de cette NOS à enseigner seraient d'être fondées sur :

- Un point de vue épistémique: la transposition de pratiques contemporaines des scientifiques ou des ingénieurs pourrait conduire à la mise à l'étude de situations authentiques et contextualisées de manières de faire de la science ;

- Un point de vue social : l'enseignement des sciences doit permettre de problématiser des questions sociales où sciences et techniques interviennent (via par exemple une approche historique ou l'analyse de controverses médiatiques) ;

- Un point de vue éthique: l'intégration des pratiques scientifiques et techniques aux grands enjeux planétaires permet de les ouvrir à des problématiques de justice sociale, de morale, etc. (Alsop et Gardner, 2017 ; Sjöström, Frerichs, Zuin, et Eilks, 2017). Elle promeut également un développement responsable des sciences et des techniques (Allchin, 2017 ; Hodson et Wong, 2017).

Parmi les différentes propositions d'actualisation de la nature de la science (par exemple Allchin, 2013 ; Kind et Osborne, 2016 ; Matthews, 2012), le modèle proposé par Erduran et Dagher (2014) a retenu notre attention. En effet, l'approche par « air de famille » (Family Resemblance Approach - FRA) nous a semblé particulièrement intéressante, car elle possède les caractéristiques attendues d'une théorie intermédiaire. Le modèle se fonde en effet sur des théories générales relatives à l'épistémologie, la sociologie, l'enseignement et l'apprentissage des sciences (Irzik \& Nola, 2014) et il se décline en un cadre théorique spécifiquement destiné à concevoir des programmes d'enseignement et des activités pédagogiques. 
C'est donc ce cadre théorique que nous développons maintenant, et que nous mobiliserons par la suite.

\section{L'approche par « air de famille » (FRA) : une théorie intermédiaire à visée didactique}

Le modèle de la nature de la science développé par Erduran et Dagher (2014) prend acte de l'impossibilité de trouver un ensemble de caractéristiques épistémologiques communes à toutes les sciences. Par contre, il s'appuie sur l'idée wittgensteinienne que les différentes sciences partagent toutes un « air de famille».

Ce modèle est constitué de trois dimensions concentriques (schéma 1).

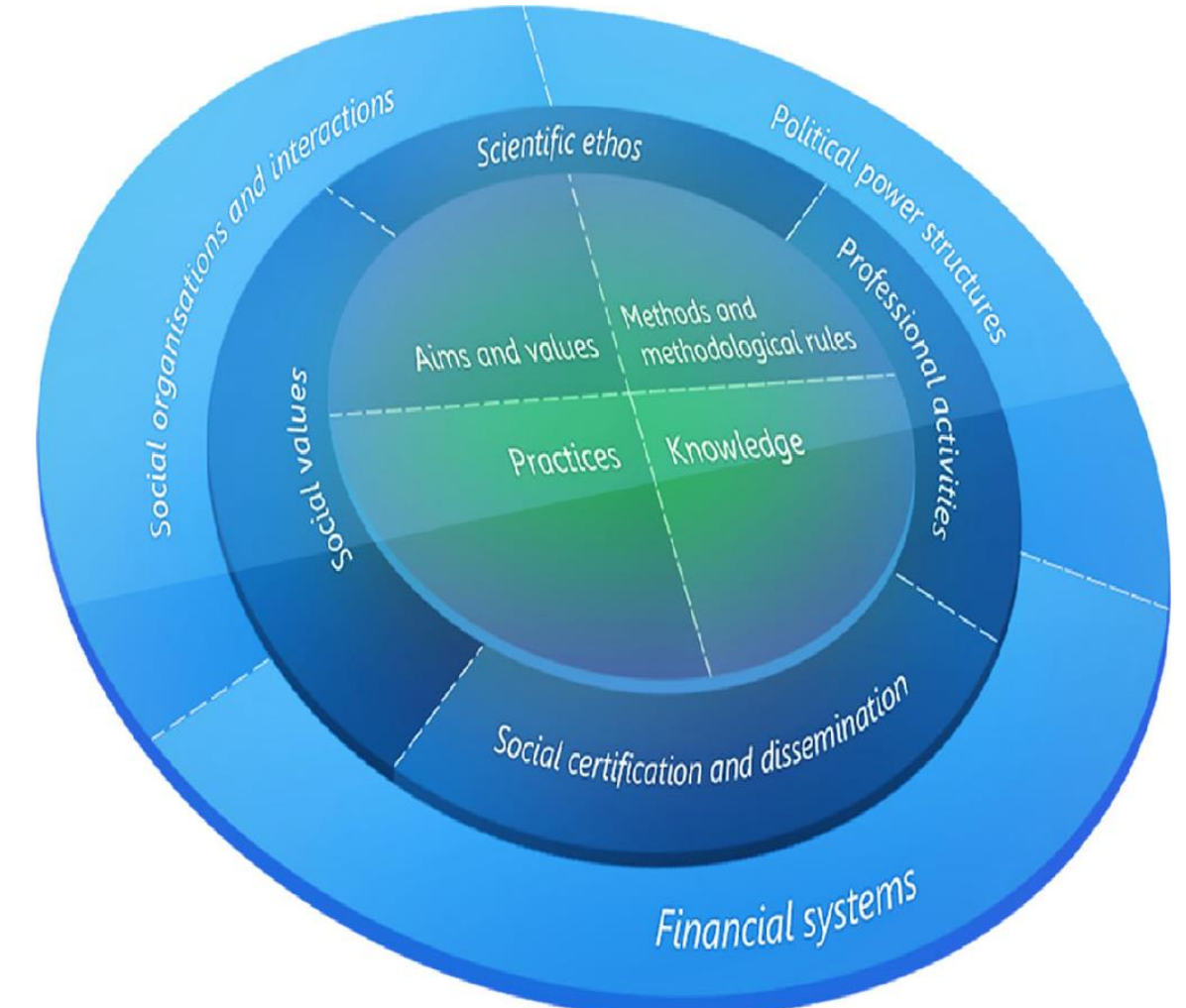

Schéma 1 : Modèle de la Nature de la Science (NOS) d’Erduran et Dagher (2014, p. 28).

Le disque central correspond à la nature épistémique de la science et est constitué de quatre catégories :

- Les savoirs scientifiques sont les produits de la recherche : lois, modèles, théories, etc.

- Les pratiques épistémiques sont les activités des chercheurs qui permettent la production et la construction d'une intelligibilité de leurs objets: expérimentation, classification, observation, c'est-à-dire plus généralement le recueil et l'analyse de données, etc.

- Les démarches d'enquête mises en place sont un point de vue plus global sur l'articulation et la justification des pratiques épistémiques.

- Les buts et valeurs font référence à la fois à des éléments axiologiques de type épistémiques (adéquation des productions avec les données, rigueur, pouvoir explicatif, objectivité, etc.) et sociaux (honnêteté, respect de l'humain, de la propriété intellectuelle).

Le disque intermédiaire correspond à la nature socio-institutionnelle de la science. II est composé de quatre catégories :

- Les activités professionnelles des chercheurs (communications, publications, recherche de fonds, expertise, etc.). 
- L'éthique scientifique correspond aux attitudes que les scientifiques sont supposés adopter dans leurs interactions avec leurs pairs ou dans la poursuite de leurs activités (pas de conflit d'intérêt, scepticisme organisé, etc.).

- Les valeurs sociales sont par exemple la liberté de recherche, le respect de l'environnement ou l'intérêt social des travaux menés.

- La certification sociale et la diffusion des résultats de la recherche font référence aux méthodes de validation et aux canaux de communication des chercheurs.

Enfin, le disque extérieur correspond à l'environnement culturel, politique, économique et social de la science, composé de trois catégories :

- L'organisation sociale de la recherche (organisation des institutions, hiérarchie, carrière, partenaires, etc.).

- Les structures de pouvoir : lien avec le politique, place des femmes, des minorités, etc.

- Le financement de la recherche et la commercialisation de ses produits.

Erduran et Dagher (2014) se servent de ce cadre théorique pour proposer une nouvelle conceptualisation de l'enseignement de la nature de la science, une analyse de curricula et un outil d'aide à la conception des enseignements.

Nous proposons d'utiliser ce modèle par la suite en tant que théorie intermédiaire pour rendre compte de l'activité de chercheurs, dans l'optique de dégager des pistes de transposition didactique.

Dans la suite de cet article, nous nous centrerons sur le domaine de l'agroécologie, car celleci constitue un axe de recherche en expansion de l'Institut national de recherche pour l'agriculture, l'alimentation et l'environnement (INRAE'1). L'agroécologie constitue un élément structurant de l'enseignement scientifique et technique dans les rénovations de diplômes de l'enseignement agricole, et elle renvoie plus largement à un questionnement sociétal sur la place des sciences et techniques dans l'agriculture et des liens avec la société. En ce sens, elle constitue un objet privilégié pour mettre en relation pratiques de scientifiques et pratiques scolaires dans un contexte scientifique et sociétal en évolution.

\section{Quelles sont les références pour enseigner l'agroécologie en lycée agricole?}

L'accompagnement de la transition de l'agriculture française vers l'agroécologie est aujourd'hui au cœur des enjeux de l'enseignement agricole (Métral et al., 2016 ; Prévost et al., 2018). Associer le concept de transition à l'agroécologie n'est pas neutre. II s'agit de souligner l'ampleur des changements à opérer et de considérer l'agroécologie non seulement comme un modèle conceptuel mais aussi comme une perspective à viser (Stassart et al., 2012). Cette transition nécessite un changement radical, tant en ce qui concerne les valeurs des acteurs, les techniques ou les connaissances scientifiques émergentes qu'ils utilisent (Rosset et Martinez-Torres, 2012).

L'agroécologie est polysémique (Reboud et Hainzelin, 2017 ; Stassart et al., 2012 ; Wezel et al., 2009), elle peut être caractérisée par deux grandes approches. D'un côté, elle s'intéresse à la conception et à la gestion des systèmes cultivés durables en mobilisant l'écologie scientifique (Gliessmann, 1998), de l'autre, elle est définie comme l'écologie des systèmes alimentaires dans leur intégralité, dans leurs dimensions écologique, économique et sociale (Francis et al., 2003).

Empreinte d'une fonction critique, l'agroécologie dénonce les pratiques des agricultures industrialisées progressivement développées après 1945 au Nord comme au Sud, les politiques agricoles qui les ont promues et les systèmes alimentaires dans lesquels l'interdépendance entre production, transformation et alimentation a éloigné le consommateur

${ }^{1}$ https://www.inrae.fr 
du producteur : un système sociotechnique très cohérent s'est structuré au fil du temps rendant très difficile l'émergence de propositions alternatives. Geels et Schot (2007) ont proposé le concept de verrouillage sociotechnique pour qualifier la situation. Ces formes d'agricultures intensives se sont fondées sur une artificialisation des milieux. Une utilisation importante d'engrais, de pesticides de synthèse et d'espèces élites sélectionnées pour leur niveau de productivité a caractérisé ces agricultures, générant des problèmes environnementaux, sanitaires, économiques, etc.

Dans le même temps, la production a été rationalisée à partir d'une approche réductionniste à la fois des métiers de l'agriculture et de la production des savoirs sur les questions posées à l'agriculture et par l'agriculture : " pour une grande part, le régime de production de savoirs scientifiques et techniques s'est structuré à partir d'objets monodisciplinaires pour produire et diffuser prioritairement des savoirs "à large portée spatiale et temporelle " (Chevassus-auLouis, 2006)2, considérés comme universels »(Cancian et Simonneaux, 2019, p. 190).

Un principe central de l'agroécologie est de favoriser une forte diversification biologique et une intensification des interactions écologiques dans les agroécosystèmes (Duru et al., 2015) afin de pouvoir substituer les processus écologiques aux intrants chimiques, de rendre les systèmes agricoles plus résilients face aux aléas et changements climatiques (Reboud et Hainzelin, 2017) et de renforcer les services écosystémiques. L'importance accordée à la biodiversité et à la diversification conduit alors, non seulement à un besoin de nouveaux savoirs en agronomie et en écologie pour mieux comprendre et modéliser les agroécosystèmes, mais aussi "d'innovations techniques, économiques, organisationnelles qui se concrétisent à l'échelle des filières » (Reboud et Hainzelin, 2017, p. 66) et qui doivent être encadrées et développées par des évolutions dans les réglementations et les politiques publiques.

L'agroécologie impulse donc un changement paradigmatique et elle est porteuse d'alternatives. Dans ce contexte, l'enseignement agricole est considéré comme un levier important pour mener les transformations du monde agricole, via la formation initiale des futurs agriculteurs, mais aussi de ceux qui encadrent la production, les techniciens et conseillers agricoles, et des enseignants qui vont les former (Direction générale de l'enseignement et de la recherche, 2014, 2020).

Cependant, la transition vers l'agroécologie est complexe, même si les systèmes agricoles intensifs et consommateurs d'intrants de synthèse sont rejetés, il n'existe pas pour autant de modèle unique sur lequel prendre appui et il convient d'aborder les changements à plusieurs niveaux pour engager le déverrouillage (Geels et Schot, 2007).

Très vite, la déclinaison opérationnelle génère des controverses qui portent notamment sur la hiérarchie des problèmes à résoudre, et par conséquent sur les solutions à favoriser. Selon Frère (2017), trois courants principaux se réclament de l'agroécologie, et contrastent suivant la priorité donnée aux dimensions suivantes:

- La dimension écologique et environnementale, en prônant par exemple la limitation du recours aux intrants de synthèse (pesticides, etc.) ;

- La dimension socio-économique, en insistant sur la nécessité de revoir les modes de distribution et d'échanges ;

- La dimension humaniste, en repensant en particulier les rapports homme - nature.

De plus Duru et al. (2015) notent de grandes incertitudes quant aux modèles scientifiques qui mettent en relation les pratiques agricoles, les processus écologiques et les services écosystémiques. Les recherches montrent également que le contexte du territoire, envisagé à la fois dans ses dimensions environnementales, économiques, sociales ou même culturelles, rend très spécifiques les savoirs et les pratiques agroécologiques, qui sont de fait difficilement transférables d'un contexte à un autre.

\footnotetext{
2 Chevassus-au-Louis, B. (2006). Refonder la recherche agronomique : leçons du passé, enjeux du siècle. Leçon inaugurale du groupe ESA, Angers, 27 septembre 2006.
} 
L'agroécologie a donc toutes les propriétés d'un problème «flou » (Fabre, 2017) : elle est "mal structurée ", puisqu'elle relève de savoirs ou de pratiques qui peuvent prendre de multiples configurations suivant les contextes ; les savoirs, moyens et finalités sont incertains ou controversés ; les dimensions du problème sont à la fois scientifiques et techniques, mais renvoient également à des choix politiques, voire éthiques; les valeurs des acteurs les conduisent à paramétrer de manière spécifique le problème dans chaque contexte.

II est donc difficile d'identifier les références sur lesquelles fonder savoirs et pratiques à enseigner car il n'y a pas de consensus sur les moyens et les voies pour décliner de manière opérationnelle l'agroécologie. Alors que le plan « Enseigner à Produire Autrement, pour les transitions et l'agroécologie " est considéré comme central pour le Ministère de l'Agriculture et de l'Alimentation, il faut bien reconnaître qu' il devance l'existence d'un jeu complet de références scientifiques et de pratiques, d'indicateurs, d'outils de pilotage et de suivi incontestables et facilement mobilisables » (Reboud et Hainzelin, 2017, p. 66). Deux éléments nous semblent particulièrement problématiques dans ce contexte pour fonder une transposition didactique des savoirs et des pratiques scientifiques de l'agroécologie : d'une part, le fait qu'il s'agisse ici d'une science en train de se faire (Latour, 1989), et d'autre part, l'enchâssement de l'agroécologie dans un objectif de transformation sociale profonde que contient l'idée de transition.

Selon Cancian et Simonneaux (2019), "la perspective agroécologique suppose d'autres façons de penser et de faire dans l'agriculture qu'il faut inventer pour renouveler les relations entre production, alimentation, santé et dignité humaine" (p. 192). C'est à la fois un changement profond dans les pratiques agricoles, les valeurs des acteurs, les modes de construction de nouveaux savoirs et de nouveaux raisonnements, et une forme renouvelée d'adhésion politique et sociale qui sont visés.

Ainsi, notre questionnement peut se formuler de la manière suivante: Qu'est-ce que les discours des chercheurs sur leurs pratiques en agroécologie peuvent nous apprendre pour envisager quelques balises curriculaires (Lange et Victor, 2006) pour l'enseignement?

\section{Méthodologie}

L'observation et l'analyse de pratiques de chercheurs sur leur lieu de travail est un objectif de nos travaux de recherche. Toutefois, cette étude exploratoire porte sur les discours des acteurs à propos de leurs pratiques en agroécologie avec comme objectif d'étudier la potentialité du cadre théorique FRA à générer des éléments structurants pour l'enseignement. A l'instar de El Hage et Ouvrier-Buffet (2018) ou bien de Wong et al. (2008), nous avons mené une série d'entretiens semi-dirigés avec des chercheurs de plusieurs disciplines académiques, à différents moments de carrière, qui travaillent tous au sein d'une même unité de recherche en agroécologie (UMR AGIR INRAE).

Plusieurs raisons ont motivé le choix de cette unité de recherche :

- Son institution d'appartenance, I'Institut national de recherche pour l'agriculture, l'alimentation et l'environnement (INRAE), est missionnée pour produire et diffuser des savoirs scientifiques sur des problématiques agricoles en lien avec les grands enjeux de société.

- L'Unité Mixte de Recherche «Agroécologie, territoires et innovations » (AGIR) développe des recherches sur les modes d'articulation entre les systèmes sociotechniques, les systèmes socio-écologiques et les systèmes agroécologiques. Son expertise scientifique est reconnue sur les questions soulevées par la transition agroécologique.

Le tableau $n^{\circ} 1$ indique les disciplines de recherche, l'expérience, et l'objet principal de recherche des différents chercheurs interrogés. 


\begin{tabular}{|c|c|c|c|c|}
\hline Agronomie & Agronomie & Économie & Agronomie & Économie \\
\hline Senior & Senior & Milieu de carrière & Milieu de carrière & Junior \\
\hline $\begin{array}{l}\text { Modélisation des } \\
\text { prises de décision } \\
\text { sur la gestion des } \\
\text { ressources }\end{array}$ & $\begin{array}{l}\text { Méta-analyse sur } \\
\text { les liens entre } \\
\text { pratiques agricoles } \\
\text { et santé humaine }\end{array}$ & $\begin{array}{lr}\text { Modalités } & \text { de } \\
\text { transition } & \text { de } \\
\text { secteurs agricoles }\end{array}$ & $\begin{array}{l}\text { Transition dans les } \\
\text { pratiques } \\
\text { d'élevage }\end{array}$ & $\begin{array}{lr}\text { Système } & \text { de } \\
\text { valeurs } & \text { des } \\
\text { agriculteurs } & \end{array}$ \\
\hline
\end{tabular}

Tableau $\mathrm{n}^{\circ} 1$ : Profil des chercheurs interrogés

Pour collecter les données, nous avons utilisé la méthode qualitative de l'entretien semi dirigé. Les entretiens ont pour objectif d'explorer plusieurs dimensions de leur activité : objets et méthodes de recherche actuels, description et évolution de leurs activités professionnelles, étapes structurantes de leur carrière, évolution des problématiques et des politiques de recherche, rapports sciences-société-agriculture, responsabilité sociale du chercheur, éléments de transmission scolaire. Ils ont été conduits dans l'institution des chercheurs. Le guide d'entretien dévolu à l'exploration de ces dimensions a été construit de façon à sonder les trois grandes catégories du modèle d'Erduran et Dagher (2014) : la nature épistémique de la science, sa nature socio-institutionnelle et son environnement culturel, politique, économique, etc. Les huit questions du guide explorant ces catégories figurent en annexe 1. Les entretiens semi-dirigés ont été conduits par un des auteurs, dans le bureau du chercheur en agroécologie. Après avoir présenté l'objectif de la recherche, les questions ont été posées dans l'ordre prévu par le guide et nous avons laissé s'exprimer librement le chercheur sans l'interrompre. Leur durée moyenne est d'une heure.

Chaque entretien a été enregistré à l'aide d'une caméra sur pied dirigée par un technicien audiovisuel de façon à garantir la qualité du son et de l'image. Les entretiens ont été intégralement retranscrits en vue de l'analyse thématique.

Une analyse catégorielle thématique de contenu (Bardin, 2013) est ensuite produite sur les corpus d'entretiens intégralement retranscrits. Elle est réalisée en deux temps : plusieurs lectures flottantes permettent de s'imprégner des données et de formuler des hypothèses de classement ; ensuite, le repérage d'indices s'effectue en fonction des catégories du cadre du modèle de la NOS d'Erduran \& Dagher (2014). Pour catégoriser les éléments de discours chaque auteur a codé indépendamment le corpus en fonction des catégories du modèle, un accord interjuge a ensuite été calculé (.81 Kappa).

Enfin, sur la base de ces catégories, nous avons comparé les discours des chercheurs de l'étude et produit une image de la nature de l'agroécologie. Ce sont ces résultats que nous présentons dans la partie suivante, et que nous illustrons à partir d'extraits de corpus.

\section{Résultats : Que disent les chercheurs en agroécologie de la nature de la science qu'ils pratiquent?}

Les résultats issus de l'analyse de contenu sont présentés en fonction des trois dimensions du modèle d'Erduran \& Dagher (2014) telles que nous les avons précédemment détaillées : la nature épistémique, socio-institutionnelle et l'environnement culturel et social de la science en agroécologie.

\section{La nature épistémique de la science en agroécologie}

\section{Les buts et valeurs}

L'objectif d'identifier et de caractériser leviers et verrous en faveur d'une transition agroécologique des systèmes de production agricole fondés sur le paradigme productiviste est un élément commun aux discours des chercheurs. Dans ce travail, comprendre et modéliser les logiques des acteurs occupent une place centrale : «pour comprendre ce verrouillage du système, il faut comprendre les logiques des acteurs à chaque niveau de la filière $(. .$.$) leurs croyances (. .$.$) leur rationalité (.$.$) et les failles dans leur système de$ 
raisonnement »(C3-3), «ça explique en partie pourquoi les systèmes sont verrouillés, parce qu'il faut s'attaquer aux valeurs, et ça c'est pas simple »(C2-24).

Deux récits se croisent dans les discours des cinq chercheurs pour donner du sens à la transition agroécologique : les agronomes mobilisent plutôt les enjeux environnementaux et sanitaires actuels, comme le changement climatique, la qualité de l'alimentation, la santé des sols, pour argumenter la nécessité de changer la manière de penser et de pratiquer l'agriculture ; les économistes inscrivent plutôt leur travail dans un récit historique et explicatif du verrouillage sociotechnique des systèmes de production agricoles, issu de choix politiques faits à la sortie de la deuxième guerre mondiale et qu'il s'agit de réorienter.

La valeur épistémique principale qui émerge des entretiens est le souci d'objectivité de leurs travaux, qu'ils fondent sur une exigence de rigueur scientifique (C1-58 « l'intérêt de la recherche c'est une espèce de rigueur méthodologique, et ça c'est un point important »), reposant sur des capacités cognitives particulières (capacités d'abstraction, de synthèse, de rédaction par exemple), comme l'expliquent $\mathrm{C} 2-8$, « je fais lire tout ce que je fais par des spécialistes des domaines qui ne sont pas le mien (...) après je réalise ce travail de synthèse en grande partie par moi-même mais je le fais corriger valider ou enrichir par d'autres collègues ", et C3-12 " on a cet objectif de rigueur c'est qu'une publication est pesée, structurée, argumentée, c'est un travail d'analyse approfondi, ce qui fait la valeur d'une publication c'est qu'elle est rigoureuse ». Les différents chercheurs soulignent également les capacités sociales nécessaires à un travail collaboratif : "recevoir la critique pour aider à avancer » (C3-31), « toute l'activité de recherche elle est essentiellement collective » (C3-4).

\section{Les savoirs produits}

Les chercheurs insistent sur l'importance de construire des savoirs qu'ils qualifient d'« intégrés ", car ils associent des corpus scientifiques de disciplines différentes (agronomie et économie par exemple), ou bien articulent des échelles descriptives différentes (par exemple l'exploitation agricole et la filière professionnelle, la santé des sols et les flux géochimiques mondiaux).

Les discours des chercheurs $\mathrm{C} 1, \mathrm{C} 4$ et $\mathrm{C} 5$ se recoupent dans l'intérêt de modéliser la prise de décision des agriculteurs en transition agroécologique, décrite à l'interface de dynamiques analysées par des sciences humaines et sociales (importance des systèmes de valeurs, description de la charge de travail, etc.) et l'agronomie (description des pratiques agricoles des agriculteurs, suivi des parcelles, gestion des ressources, etc.). De même, C3 analyse les processus de transition de la filière des légumineuses à graines, en mettant en relation les différentes échelles de production, transformation et consommation des produits agricoles.

C2 travaille également à produire un savoir intégré du lien entre pratiques agricoles et santé humaine, par la mise en relation et la hiérarchisation de savoirs multidisciplinaires.

Les savoirs ont aussi pour vocation de répondre aux grands enjeux planétaires : "quand on a une vision systémique multi-enjeux c'est pas que l'agriculture et l'alimentation mais à la fois derrière il y a le changement climatique, il y a la déforestation, il y a le bien-être animal, quand on couple tous ces enjeux-là finalement on s'aperçoit que ça aide à hiérarchiser les politiques publiques et peut-être les thèmes de recherche à développer »(C2-30), "si on n'avait pas une approche systémique intégrée on ne pouvait apporter aucune recommandation auprès du politique »(C3-8).

Ces différents travaux ont ainsi l'ambition d'identifier des points-clés pour le déverrouillage des trajectoires des systèmes sociotechniques et les possibilités d'articulation des solutions à différents niveaux des transitions.

\section{Les pratiques épistémiques}

Les pratiques sont très diverses et dépendent bien évidemment de l'ancrage disciplinaire des chercheurs. Ainsi, le travail empirique des agronomes se caractérise par la mise en œuvre d'expérimentations et/ou de modélisations/simulations alors que les chercheurs en économie travaillent davantage à partir d'entretiens et de bases de données statistiques.

L'interdisciplinarité est centrale dans les discours des différents chercheurs: tous les chercheurs la considèrent comme une pratique significative de leur activité, et l'animation 
scientifique de l'Unité y contribue, " on fait vivre l'interdisciplinarité par des projets interéquipes [...] et par des animations de séminaires 》 (C1-54).

Les chercheurs ont toutefois un point de vue nuancé sur l'interdisciplinarité suivant leur affiliation disciplinaire. En effet, les agronomes considèrent que les sciences humaines et sociales (SHS) leur ont permis de problématiser des questions jusque-là hors de leur cadre de recherche : «il y avait pas mal de questions qui venaient des sciences sociales qui étaient posées par la société et qui n'étaient pas posées par les chercheurs » (C1-18), " on a sur cette phase de transition agroécologique principalement des travaux en SHS et on a très peu travaillé en agronomie, en agronomie traditionnellement on préfère des systèmes stabilisés » (C4-8), « le travail que j'ai développé sur les systèmes alimentaires (...) il a trouvé sa légitimité aussi parce qu'il était cohérent avec des thématiques qu'on mettait en avant dans les sciences sociales » (C2-14). Cette interdisciplinarité conduit à la définition de problématisations partagées, notamment du fait de la nécessité de travailler sur des "chaînons manquants " entre champs de recherche agronomique et champs couverts par les SHS (C2 développe dans l'entretien son travail consistant à associer les savoirs sur l'alimentation animale et ceux sur la santé humaine).

Du côté des SHS, on note l'intérêt de l'interdisciplinarité pour s'acculturer aux problématiques agricoles pour des scientifiques dont la formation est souvent éloignée de l'agriculture. L'interdisciplinarité leur permet donc de prendre pour objet de recherche un domaine non familier, mais sécurisé par la proximité des agronomes : « je ne connaissais rien à l'agriculture (...) il a fallu que je discute beaucoup avec les agronomes » (C3-8), «j'avais jamais travaillé sur l'agriculture avant et donc voilà j'ai appris énormément en travaillant avec eux sur ce papier-là bon c'est pour ça que je dis c'est une matrice pour moi ça cadre ma manière de penser les relations entre agriculture et environnement $»(\mathrm{C} 5-14)$.

\section{Les démarches d'enquête}

L'interdisciplinarité et les recherches participatives sont deux éléments structurant les démarches d'enquête des chercheurs.

L'interdisciplinarité est conçue comme favorisant la production d'un savoir "intégré ", notamment parce qu'elle oblige à " hiérarchiser, mettre en relation et identifier des liens majeurs qui sont sources de nouvelles connaissances » (C2-6). C4-10 déclare ainsi que le travail « en collaboration avec les chercheurs SHS (...) permet d'hybrider [les] représentations et d'arriver à une production scientifique ". C5-14 précise la manière dont sciences agronomiques et SHS se combinent à partir d'un exemple de collaboration : « nos méthodes étaient ce que produisaient les agronomes, et inversement (...) ce qu'ils utilisaient en méthode c'était ce qu'on produisait nous c'est-à-dire l'analyse sociale, socio-économique de ces formes d'agriculture ». L'interdisciplinarité agit dans ce cas comme un moyen de circulation des méthodes et des résultats : le travail des uns intègre les éléments des autres pour produire des résultats originaux.

Les chercheurs pointent la nécessité de la démarche interdisciplinaire, au vu de la complexité des objets de recherche : « on a besoin de l'enrichissement de ces différentes disciplines pour comprendre le comportement des acteurs (...) un agriculteur va raisonner d'une certaine façon, bon je vous dis des mécanismes prix quantité pour l'économie ça reste fondamental, mais il y a pas que, il y a aussi ses connaissances agronomiques, ses connaissances techniques qui va faire qu'il agit d'une certaine façon, donc ça ce sont les sciences agronomiques qui vont nous aider à le comprendre »(C3-8). Ainsi, du fait de la collaboration avec les SHS, les agronomes ont pu « développer des concepts et des méthodes qui permettent d'appréhender la dynamique des systèmes et donc d'avoir une appréhension de la durabilité qui ne soit non plus une photo à l'instant $t$ mais une représentation d'une trajectoire » (C4-8). L'interdisciplinarité conduit à « voir le monde dans un kaléidoscope » (C158), à rediscuter de concepts stabilisés dans un champ scientifique contribuant à favoriser l'appropriation pour une problématisation à plusieurs mains : «le savoir c'est un acquis validé par les pairs mais comme en fonction des disciplines les pairs ne sont pas les mêmes c'est un des grands intérêts [...] pas mal de questions peuvent se reposer, c'est quoi un territoire ? c'est quoi un animal ? » (C1-58). 
Les thématiques explorées par les chercheurs rendent également nécessaires des approches de type participatives (C1-20: " nous on le mobilise énormément parce que je pense que sur les thématiques sur lesquelles on est on en a besoin »). C2-22 développe par exemple le cas d'une recherche sur les critères de performance de systèmes agricoles, qui ont été co-définis avec les agriculteurs et éleveurs: " c'est un peu un fil rouge de la recherche que j'ai fait toujours avec des agriculteurs et des éleveurs qui étaient à la fois (...) sujets et objets de la recherche ". De même, C3-14 déclare l'importance de la visée transformative de ces recherches: "si vous co-concevez les solutions de demain avec les acteurs qui vont les mettre en œuvre on peut espérer que ça va être mis en œuvre plus rapidement et plus efficacement ». Ces démarches résonnent également avec les valeurs explicitées par certains chercheurs : « il est clair que le fait d'apprécier les méthodes participatives c'est parce que moi je considère et en chercheur et en citoyen qu'il est important de mixer les mondes si on veut trouver une solution » (C1-34).

\section{La nature socio-institutionnelle de la science en agroécologie}

\section{Valeurs sociales et éthique professionnelle}

La transition des modes de production agricole et des systèmes alimentaires fondés sur des principes agroécologiques est une valeur forte pour les chercheurs interrogés. Leur éthique scientifique est corrélée aux valeurs sociales qu'ils attribuent à leurs recherches. En effet, leurs objets de recherche sont choisis pour correspondre à une éthique de ce qu'ils considèrent comme être la « vie bonne », soucieuse de l'environnement, de la santé et de la justice sociale. C5-22 considère ainsi " qu'il y a au moins une dimension morale qui intervient dans les choix qu'on fait dans les questions de recherche (...) je travaille pour le bien commun (...) parce que je considère que le développement durable est un bien et la poursuite de cet objectif améliore le bien-être de l'humanité ».

Leurs recherches visent une amélioration du bien commun et « l'utilisation parcimonieuse des ressources » (C1-34), notamment parce qu'elles les rendent sensibles aux enjeux de durabilité de l'agriculture: " tous les problèmes de santé publique qui sont aujourd'hui associés à l'agriculture (...) plus ou moins liés avec l'exposition aux pesticides, je suis assez convaincu sur la base de tous ces éléments là qu'il y a nécessité d'une transition (...) peu de viande, maîtrise du carbone, des phytos " (C4-40), " les problèmes sont graves on va dans le mur à la fois pour la santé humaine, on vit moins longtemps en bonne santé (...) et en même temps on va dans le mur parce qu'on consomme trop de ressources (...) »(C2-18), « réorienter cette trajectoire vers des systèmes agroalimentaires plus durables (...) avoir une alimentation moins riche, une pression moindre sur les écosystèmes » (C3-2). Le travail de recherche participe également à l'évolution dans leur propre point de vue sur les questions qu'ils étudient : «je suis quelqu'un qui, en ayant compris ces enjeux, j'ai inséré plus de légumineuses dans mon alimentation au quotidien, ma famille est sensibilisée, (...) le chercheur peut aussi avoir cet acte de démonstration dans son entourage des valeurs qu'il défend »(C3-8), « je pense que j'y suis déjà, j'ai plus de lentilles dans mon assiette que de produits carnés donc (...) je mets en phase mes valeurs de citoyen avec mon projet professionnel » (C4-38), « je deviens plus que convaincu et deviens presque un militant, non pas... mais au sens... avec le débat scientifique » (C2-10).

Cette volonté de prendre part à la transition par leurs recherches fait qu'ils inscrivent leur action auprès de différents publics : agriculteurs, consommateurs, citoyens, politiques, mais aussi chez les collègues scientifiques pour promouvoir l'étude de thèmes en lien avec les grands enjeux.

\section{Certification et diffusion des produits de la recherche}

La certification des savoirs produits obéit à des règles usuelles de fonctionnement scientifique: évaluation par les pairs, publication dans des revues scientifiques, communications scientifiques. C2 souligne une évolution vers davantage de publications dont la qualité tient à leur nature intégrative plutôt qu'analytique. 
Les chercheurs font état également de l'importance d'une diffusion au-delà du public académique: rendre compte des recherches auprès des agriculteurs (C4-12 «j'attache beaucoup d'importance à faire des restitutions aux collectifs d'éleveurs qui travaillent avec nous »), du grand public (pour sensibiliser les citoyens aux enjeux d'agriculture et de santé, $\mathrm{C} 2$ ), des politiques (pour favoriser les prises de décision politique, C3) et auprès d'autres chercheurs (pour travailler l'épistémologie de la discipline C5-22), afin de faire avancer la prise en compte des questions liant agriculture - environnement et santé humaine dans la société.

\section{Activités professionnelles}

Cette volonté de participer à la transition par leurs recherches complexifie le métier de chercheur, et leur travail se structure en de multiples activités : recherche, valorisation, gestion des projets, missions d'expertise, administration, partenariats.

Parmi leurs activités professionnelles, les chercheurs en distinguent certaines : C5 insiste sur la pression à la publication (C5-26 « il y a quand même des pressions par rapport à tout ce climat d'évaluation de la recherche »), qui le conduit à ne pas prendre le temps de se documenter comme il le souhaiterait, au contraire de C2 qui, en fin de carrière, prend le temps de frayer et de faire des synthèses bibliographiques interdisciplinaires. C1 dirige l'unité de recherche et évalue à $60 \%$ de son temps la gestion administrative de l'unité et le management de ses personnels, auquel s'ajoute un temps d'animation scientifique d'interface avec l'institution nationale. C3 met l'accent sur l'organisation de rencontres avec les professionnels (les agro-industriels) et la communication, afin de sensibiliser à de nouvelles espèces à produire (les légumineuses). Ces préoccupations recoupent les trajectoires de carrière de ces chercheurs, focalisés plutôt sur le stress de la publication en début de carrière, l'animation de réseaux ou de collectifs en milieu de carrière, et puis de la communication libérée des enjeux professionnels en fin de carrière.

\section{L'environnement social de la science en agroécologie}

\section{L'organisation sociale de la recherche}

Les chercheurs interrogés trouvent dans l'unité de recherche des ressources pour travailler leurs objets, à la fois pour mener à bien les projets collectifs, mais aussi pour construire une expertise au niveau de l'unité : "avoir une production collective qui fasse ressortir des originalités qui sont travaillées dans l'équipe » (C3-11).

L'animation de séminaires communs rend possible le travail interdisciplinaire : " on a investi dans des séminaires hebdomadaires pour que les économistes comprennent les agronomes et réciproquement et ça a été payant »(C2-16). Ce travail s'est ainsi structuré autour de cadres d'analyse susceptibles de mettre en interface les différentes approches (comme par exemple les concepts de systèmes socio-écologiques et sociotechniques). Le co-encadrement de doctorants (C4-C5), l'écriture de projets conjoints, l'organisation de séminaires dédiés sont autant d'espaces qui permettent le développement institutionnel de l'unité et l'enrichissement de ses thématiques de recherche $(\mathrm{C} 1)$.

Cette manière interdisciplinaire de travailler est originale selon les chercheurs, mais correspond cependant à des orientations stratégiques de I'INRAE (C4 cite les " métaprogrammes » de l'INRAE) visant à explorer les liens entre agriculture, environnement et alimentation. L'INRAE participe également aux débats sociétaux et politiques, en particulier par la rédaction de documents d'expertise : " l'INRAE répond à des commandes du ministère pour faire des expertises collectives (...) c'est un travail qui s'étend sur 2, 3, 4 ans qui mobilise énormément de chercheurs (...) c'est un travail sérieux et qui surtout approche les problèmes avec une certaine globalité » (C2-20).

C1-24 souligne une demande plus pressante de l'institution pour davantage intégrer les recherches dans un système favorisant la valorisation des productions scientifiques dans les projets avec " la mise en place d'une délégation partenariat-transfert-innovations avec des chargés d'innovation sur 7 à 8 thèmes principaux qui touchent l'INRAE ».

Une partie de l'activité professionnelle des chercheurs consiste à faire vivre différents réseaux d'acteurs : des instituts techniques (Institut de technologie agroalimentaire, Institut technique 
du végétal, etc.), des acteurs agro-industriels ou des opérateurs de filière, des collectivités publiques, des syndicats ou associations, des chercheurs étrangers (" l'intérêt du côté européen c'est d'offrir des possibilités de généralisation qui sont plus importantes et d'avoir des contextes un peu différents pour se poser les questions de manière plus robuste ", C428), et bien sûr des agriculteurs.

\section{Les structures de pouvoir}

Les chercheurs développent dans les entretiens les liens entre politique et science.

C3 et C5 justifient la rigueur de leur recherche, pour forger notamment des arguments susceptibles de convaincre les décideurs de lever les verrouillages : «il faut justifier encore plus scientifiquement qu'il faut changer tel ou tel levier, il faut encore travailler avant que les politiques entreprennent des actions »(C3-10), «il faut gagner sa légitimité en tant que scientifique pour avoir une certaine visibilité politique »(C5-20). C2 et C3 participent à des missions d'expertise, qu'ils considèrent comme une interface entre science et politique : « tout le lobbying que le chercheur doit faire pour convaincre les financeurs de l'intérêt de conduire un travail de recherche précis »(C3-12), « il faut arriver à leur [les politiques] faire comprendre, toucher du doigt ce problème pour qu'ils aient bien conscience que le scientifique leur permet de justifier leur action » (C3-6).

Pour ces chercheurs, la science est un service public dont les résultats doivent être accessibles gratuitement et facilement, c'est pourquoi ils s'engagent dans des recherches participatives et valorisent la diffusion de leurs travaux au-delà de leurs pairs, avec l'idée que c'est au travers « de niches d'innovation dans des petits réseaux d'acteurs plus ouverts sur l'innovation radicale que le réseau dominant va être bousculé et changé » (C3-10).

\section{Le financement de la recherche et la commercialisation de ses produits}

Les chercheurs mentionnent également le verrouillage de la recherche sur leurs thématiques : " l'essentiel des fonds de recherche va pour une agriculture qui n'est pas agroécologique qui va vers une agriculture durable certes mais durable au sens où on contrôle ce qui rentre ce qui sort avec des nouvelles technologies (...) et l'essentiel de la recherche est faite là et les fonds vont là dans le monde européen »(C2-40).

La rédaction de réponses à des appels à projets est aussi devenue la norme pour financer la recherche (C4-19 « aujourd'hui quelqu'un qui n'aurait pas de projet de recherche ne pourrait pas subvenir à ses besoins de fonctionnement »), elle est considérée comme servant à piloter la recherche par les grands enjeux définis par le ministère de l'agriculture, sans rogner sur la liberté des chercheurs.

\section{Perspectives pour une transposition didactique}

\section{Points saillants de la nature de la science en agroécologie}

Nous retenons quatre éléments de la nature de la science en agroécologie à partir de nos résultats.

Premièrement, ce qui ressort massivement des entretiens est la nécessité d'un regard entremêlé des disciplines pour la construction de savoirs sur la transition agroécologique. Bien plus qu'une multidisciplinarité qui conçoit le travail côte à côte de chercheurs de disciplines différentes, c'est bien de transdisciplinarité (Claverie, 2010) que nous parlent ces chercheurs. Au-delà des polémiques de l'acceptabilité (Gendron, 2014), l'enjeu est d'articuler, sans instrumentaliser, les humanités et les sciences agronomiques pour co-construire des savoirs hybrides et des méthodologies plurielles pour une nouvelle intelligibilité des processus de transition. Le contrôle de la validité des résultats est ainsi assuré par la confrontation d'épistémologies différentes.

Le deuxième point saillant, est l'approche inductive revendiquée par les chercheurs. C'est une recherche pragmatique à partir d'études de cas qui motive leur travail. Pour chacun d'eux, il convient de partir du terrain, de ce qu'ils nomment les « niches », les «acteurs », les 
"territoires », afin de co-comprendre les leviers et verrous dans la mise en œuvre de la transition agroécologique. Cette recherche pragmatique peut être qualifiée de recherche intégrative dans le sens où elle englobe les acteurs, les savoirs, de façon à rendre productive une intelligence collective capable de construire des récits systémiques et porteurs de sens.

Le troisième point saillant est la cohérence entre les valeurs personnelles et les valeurs de recherche dont témoigne chaque chercheur. Bien entendu le sujet de recherche s'y prête, tous travaillent au service de la transition agroécologique et du changement de paradigme de production agricole. Toutefois, si de nombreux chercheurs sont convaincus de l'intérêt de leur travail de recherche, tous ne modifient pas pour autant leurs agirs personnels. Les valeurs écocitoyennes sont ici un puissant moteur et questionnent les rapports entre subjectivité et objectivité de l'activité scientifique. Ces chercheurs engagés nous disent qu'ils ne pourraient pas travailler sur des sujets de recherche qui ne correspondent pas à leurs valeurs, donc que leur choix de recherche est empreint de subjectivité, tout en insistant tous sur l'obligatoire rigueur scientifique à mettre en œuvre pour convaincre les professionnels, les politiques et attester de la qualité de leur travail de recherche.

Le quatrième point saillant est la responsabilité épistémique qu'ils attribuent à leur rôle social : la pertinence des savoirs construits est évaluée à leur capacité à répondre aux grands enjeux du développement humain du $21^{\text {ème }}$ siècle (climatiques, sanitaires, alimentaires, etc.) et à transformer les courants de pensée et d'action dominants, aussi bien au niveau de l'épistémologie des disciplines, qu'au niveau des pratiques scientifiques et agricoles, ou bien qu'à celui des financements et des politiques publiques.

\section{Quelles pistes de transposition pour la classe dans le cadre de la didactique des QSV ?}

L'agroécologie irrigue tous les référentiels de formation de l'enseignement agricole, quel que soit le niveau d'apprentissage (du cycle 4 au BTSA) et les filières de la formation (dont les spécialités sont très diverses : services à la personne, conduite et gestion d'une entreprise agricole, gestion des espaces, génie alimentaire, etc.). De plus, nous avons vu que l'ancrage territorial de l'agroécologie fait que les savoirs et pratiques construits sont difficilement transférables d'un contexte à un autre.

Aussi, ce sont non seulement les parcours de formation des élèves qu'il nous faut prendre en compte, mais également la grande diversité des territoires dans lesquels se situent les lycées agricoles, qui font que l'agroécologie y prend nécessairement une forme singulière.

Comment envisager un processus de transposition didactique dans ces conditions?

II nous semble que notre étude dégage trois principes structurants, qui permettent à la fois de constituer des balises communes pour l'ensemble des référentiels, publics et territoires, et sont également susceptibles d'être déclinées et mises en œuvre localement.

\section{Balise 1 « la science compliquée »- Enseigner les liens entre les activités humaines et les conséquences environnementales et sanitaires dans le domaine de l'agriculture}

Le premier principe est que les savoirs et pratiques agroécologiques sont empreints d'incertitudes alors que le diagnostic qui les légitime devient dominant et plus documenté. Les enjeux et les données associant les problématiques environnementales, sanitaires et sociales aux questions agricoles (changement climatique, érosion de la biodiversité, dégradation des écosystèmes et de la santé humaine, inégalités sociales, mondialisation de l'économie, etc.) constituent le ressort qui pousse les chercheurs à aligner leurs valeurs personnelles et les valeurs de la recherche.

Une première balise consiste ainsi à rendre compte de la réalité des processus irréversibles solidement étayés par la recherche : ils font généralement l'objet de rapports collectifs ou institutionnels synthétisant l'état des savoirs sur la question, en s'efforçant de constituer une 
perspective intégrée et multi-échelles (comme le font les rapports du $\mathrm{GIEC}^{3}$, de I'IPBES ${ }^{4}$, ou des instituts scientifiques tels l'INRAE). Le consensus scientifique sur ces phénomènes permet de problématiser les enjeux de la production agricole, des systèmes alimentaires ou des habitudes de consommation, et plus généralement permet de discuter les conséquences des activités humaines sur l'environnement. Cette manière d'envisager les sciences et les techniques est ce que Coutellec (2015) appelle la " science compliquée », c'est-à-dire la science " hyperspécialisée, travaillant sur des problèmes hypercomplexes » (p. 56). S'il est important d'enseigner aux élèves l'état de la science sur ces grandes questions afin d'en faire des enjeux engageant l'avenir, il est nécessaire de compléter cette image de la science par deux autres aspects : la science « expliquée » et «impliquée ». La science expliquée permet de légitimer et donner confiance en l'activité scientifique et technique, en rendant intelligible la manière dont ces savoirs sont construits. De même, la " science impliquée » (Coutellec, 2015) valorise ses dimensions sociales, citoyennes, participatives.

\section{Balise 2 « la science expliquée » - Enseigner explicitement la nature de la science en agroécologie}

Expliquer la science en tant qu'activité sociale est ainsi un deuxième principe. L'enseignement de l'agroécologie gagnerait à concevoir des situations d'enseignement apprentissage qui transposeraient les éléments de la nature de la science que nous avons précédemment décrits.

Donner à voir l'activité scientifique du chercheur en l'inscrivant dans sa dynamique collective (au sein de laboratoires ou d'équipes), en interaction avec d'autres acteurs sociaux peut contribuer à valoriser son expertise. En effet, montrer la mixité des approches, la multiplicité des espaces de travail, et la vigilance assurée par la confrontation et les modes de validation d'épistémologies différentes peut être une piste pour donner confiance en l'intelligence collective que déploient les chercheurs. A ce titre, la littérature "grise » de vulgarisation produite par les chercheurs et adressée à un public averti non spécialiste peut constituer des ressources intéressantes pour l'enseignement. De même, les entretiens de chercheurs que nous avons filmés, ou bien que les élèves pourraient eux-mêmes mener, offrent des possibilités pour travailler des éléments spécifiques de la nature de la science en agroécologie. Pour cela, le cadre théorique FRA a le mérite de pointer certaines dimensions que les enseignants pourraient aborder avec leurs élèves (par exemple l'intérêt des démarches interdisciplinaires et ses obstacles, les modes de financement ou de certification, la science participative, la place des valeurs, etc.).

\section{Balise 3 «la science impliquée» - Situer la démarche d'enquête au cœur de l'enseignement}

Le troisième principe est la place centrale à accorder au problème et au processus de problématisation dans les situations d'enseignement-apprentissage (Fabre, 2009, 2011). En effet, pour articuler des enjeux variés et définir des objectifs à atteindre, de nombreuses configurations agroécologiques sont possibles pour apporter des réponses à un problème. II convient non seulement de les identifier, mais aussi de discuter et/ou de choisir la solution la plus à même d'être partagée par les parties prenantes. En d'autres termes, mettre en place des pratiques agroécologiques, c'est construire le cadre d'un problème dans lequel vont se combiner des éléments empiriques, nécessairement situés, et des savoirs scientifiques et techniques (Duru et al., 2015), dont la pertinence va être continuellement évaluée au regard d'objectifs multidimensionnels (écologiques, agronomiques, économiques, éthiques, etc.).

La problématisation en tant que processus d'enseignement-apprentissage engage donc un travail avec les élèves sur l'analyse des spécificités d'un contexte local, l'identification des savoirs scientifiques et techniques mobilisables en situation, la discussion et la clarification de visées plurielles. Métral et al. (2016) ont montré combien il était difficile d'enseigner des savoirs

\footnotetext{
${ }^{3}$ Groupe d'experts intergouvernemental sur l'évolution du climat

4 Plateforme intergouvernementale scientifique et politique sur la biodiversité et les services écosystémiques (Intergovernmental Science-Policy Platform on Biodiversity and Ecosystem Services)
} 
agroécologiques parce que ceux-ci sont susceptibles d'être «disqualifiés » par l'environnement familial des élèves ou dans leurs lieux de stage. Ainsi, la résistance des acteurs des territoires aux changements trop éloignés de leur système de valeurs et de références peut constituer un élément problématique à considérer, davantage en tant que condition ou donnée du problème à résoudre plutôt qu'en tant qu'obstacle à l'apprentissage de savoirs agroécologiques.

La manière dont les chercheurs construisent le cadre des problèmes qu'ils étudient est à ce titre intéressant car comprendre les leviers et freins à la transition agroécologique est justement un objet de leurs recherches.

Ainsi, le caractère participatif des pratiques de recherche contextualisées à des études de cas est revendiqué par cette équipe de chercheurs, ce qui permet aux différents acteurs (scientifiques, agriculteurs, etc.) de s'impliquer dans la définition des problèmes, la mise en place des méthodes et l'interprétation des résultats. Travailler des études de cas, en étudier le processus de problématisation opéré par des acteurs y participant, permettrait aux élèves de comprendre comment le questionnement scientifique se construit avec les acteurs sociaux en lien avec les problèmes locaux.

Les entretiens des chercheurs nous montrent également que leur engagement scientifique est enchâssé dans un engagement social et politique, ce qui les force à la fois à objectiver leurs objets et méthodes d'une manière spécifique et à assumer un rôle social. La construction d'un problème scientifique est ainsi mis en relation avec un problème social et politique. Une piste d'enseignement consiste donc à mettre en avant et en discussion dans l'école cette nature engagée.

Ainsi, une troisième balise pour l'enseignement se dégage : problématiser des pratiques agroécologiques dans la mesure où une transition agroécologique impose des modalités d'enseignement spécifiques.

On note ici une certaine résonance entre ce qui émerge des discours des chercheurs et un modèle de démarche pédagogique, appelé démarche d'enquête, proposé pour l'enseignement des QSV (Simonneaux et al., 2017). Le modèle de la démarche d'enquête est né d'un rapprochement entre l'étude scolaire des QSV et les démarches d'investigation scientifique avec comme objectif la construction de savoirs socio-scientifiques incertains. Nos résultats nous incitent donc à étudier le statut de la démarche d'enquête dans la didactique des QSV, non seulement pour «orchestrer » des dispositifs pédagogiques visant la construction de savoirs scientifiques et techniques, mais au-delà, comme une démarche intégrant certaines particularités des pratiques des chercheurs, ici en agroécologie.

La démarche d'enquête sur une QSV est conçue comme une succession de cinq phases, pouvant se répéter, en fonction du projet d'enseignement, et permettant la problématisation de la question au fil de l'enquête : le recueil et l'analyse d'informations, la réflexivité/subjectivité des enquêteurs, l'explicitation et la construction des raisonnements, les réponses possibles et actions conduites, le compte-rendu de l'enquête.

II n'y a pas d'ordre prédéfini à suivre, mais une condition dans la démarche est que les différentes phases doivent toutes être mises en œuvre dans le dispositif pédagogique.

Elles sont exemplifiées dans le tableau $n^{\circ} 2$. 


\begin{tabular}{|c|l|}
\hline $\begin{array}{c}\text { Dimensions de la démarche } \\
\text { d'enquête }\end{array}$ & \multicolumn{1}{|c|}{ Exemples } \\
\hline $\begin{array}{c}\text { Recueil et analyse } \\
\text { d'informations }\end{array}$ & $\begin{array}{l}\text { Nature des données : scientifiques, syndicales, juridiques... } \\
\text { Nature des sources : enquête de proximité, interview d'expert, médias Web, } \\
\text { discours d'acteurs } \\
\text { Acteurs impliqués : visibles/cachés } \\
\text { Nature des arguments : scientifique, axiologique, économique, politique... } \\
\text { Critique des informations : fiabilité, redondance, pertinence... ou } \\
\text { opposition }\end{array}$ \\
\hline $\begin{array}{c}\text { Réflexivité / subjectivité des } \\
\text { enquêteurs }\end{array}$ & $\begin{array}{l}\text { Réflexivité / subjectivité : Distance des enquêteurs face au problème et aux } \\
\text { acteurs }\end{array}$ \\
\hline $\begin{array}{c}\text { Explicitation et construction } \\
\text { des raisonnements }\end{array}$ & $\begin{array}{l}\text { Mise en relation des données / des acteurs / des actants (conjectures) } \\
\text { Explicitation des raisonnements socio-scientifiques } \\
\text { Identification des nouds de controverse } \\
\text { Construction, mobilisation d'un / de modèle(s) }\end{array}$ \\
\hline $\begin{array}{l}\text { Ientification des incertitudes, des risques... } \\
\text { Pistes de réponses possibles }\end{array}$ & $\begin{array}{l}\text { Proposition d'actions possibles : politiques et citoyennes, scientifiques, } \\
\text { professionnelles... locales (dans le territoire, dans le lycée...) ou globales }\end{array}$ \\
\hline $\begin{array}{c}\text { Rendre compte de la } \\
\text { démarche et des conclusions } \\
\text { provisoires de l'enquête }\end{array}$ & $\begin{array}{l}\text { Rendre public la démarche, la (les) solution(s), les positions, les arguments, } \\
\text { les actions possibles..., la problématisation et son évolution, les modes } \\
\text { d'organisation du travail, les temps de régulation, les supports de travail, } \\
\text { les dysfonctionnements }\end{array}$ \\
\hline
\end{tabular}

Tableau 2 : Illustration des différentes phases de l'enquête (à partir de Simonneaux et al., 2017, p. 150).

La démarche d'enquête peut ainsi permettre d'organiser la mise en activités des élèves, afin de leur permettre d'étudier un problème local en lien avec la transition agroécologique.

Les caractères participatifs et d'engagement social et politique de la science relevée dans les entretiens peuvent ici être repris. En effet, le problème soumis aux élèves peut être authentique, négocié avec des partenaires extérieurs, afin qu'ils proposent non seulement une trajectoire sociotechnique de transition, mais aussi un accompagnement à la transition.

L'enquête peut les conduire à mettre en œuvre une pluralité de méthodes (issues des sciences et techniques et/ou des sciences humaines et sociales) qui rend compte de la pertinence d'une diversité de savoirs, aussi bien disciplinaires que d'expérience, pour appréhender le problème. L'articulation de cette diversité nécessite une prise en charge didactique de nature interdisciplinaire. De plus, les savoirs construits par les élèves sont orientés vers la réalisation d'une action, qui fait de l'action éducative une action sur et pour le territoire. Enfin, l'explicitation des valeurs, leur négociation au sein d'espaces didactiques spécifiques (comme les débats) conduit à considérer les valeurs et émotions individuelles comme un élément interne au problème à résoudre, qui peut être de nature éthique ou affectif.

L'ensemble de ces éléments font que l'on peut qualifier cette approche de "science impliquée ", entendu comme la reconnaissance que l'agroécologie est une science fondamentalement intriquée, tissée au réel aussi bien naturel que social (Coutellec, 2015).

\section{Limites de l'étude}

Mener des entretiens semi-dirigés avec des chercheurs d'une même équipe a l'avantage d'explorer les dynamiques collectives d'une communauté restreinte de recherche, les habitudes de travail entre collègues, les objets de travail commun, les valeurs partagées. Finalement, mener une analyse à l'échelle " micro » de la science en train de se faire (Simonneaux, 2017) relève peut-être autant de la description d'un contexte spécifique de travail que d'une nature de la science. Autrement dit, si l'étude prise au niveau de l'équipe de recherche permet de gagner en cohérence sur le fonctionnement « local » de la recherche, la diversité des perspectives sur la question s'efface. C'est pourquoi, de notre point de vue, le fait de documenter les pratiques des scientifiques ne se substitue pas à une analyse socioépistémologique de la question socialement vive, elle constitue une pièce méthodologique 
complémentaire et contribue à construire les références des savoirs et des pratiques à enseigner.

De même, les techniques d'entretien permettent d'accéder à ce que les acteurs disent de leurs pratiques, et pas directement à leurs pratiques. Ainsi, il serait intéressant comme l'a fait par exemple Gardes (2013) d'observer directement les pratiques de recherche. Nous reviendrons sur ce point dans la conclusion.

Le cadre de l'approche par « air de famille » nous semble une théorie intermédiaire sur la NOS intéressante. Elle offre un cadre général permettant une vue systémique sur la nature de la science, aussi bien d'un point de vue strictement épistémologique que sociologique. II pourrait être intéressant de la coupler à des cadres plus spécifiques comme celui mobilisé par exemple par El Hage \& Ouvrier-Buffet (2018). Cela aurait l'avantage de multiplier les échelles de comparaison: nature des sciences mises en jeu et focale sur certaines dimensions. On retrouve d'ailleurs certains résultats similaires, notamment sur l'intérêt qu'éprouvent les chercheurs à changer de cadres d'analyse ou de modèles afin de transformer le problème qu'ils ont à résoudre. On conçoit donc que l'analyse de pratiques de scientifiques peut rejoindre ici les recherches didactiques sur la problématisation.

\section{Conclusion}

Les réformes en cours dans l'enseignement agricole visent à déployer massivement l'enseignement de l'agroécologie, sans que les références de ce qu'il y a à enseigner soient pour autant explicitées par l'institution scolaire.

Nous montrons dans cette étude que ces enseignements gagneraient à intégrer des discours de chercheurs, en particulier pour donner voix à leur sensibilité, leur attachement à leur objet de recherche, leur engagement social. La nature de la science ainsi décrite est intrinsèquement humaniste, au service d'un projet d'habitabilité du monde pour les humains et non humains, un projet de science pragmatique, qui relie savoirs, personnes, collectifs, et les territoires, actuels et futurs, dans lesquels ils vivent. Autrement dit, cette manière d'être du scientifique exige une transposition didactique au service de la construction d'une pensée complexe (Morin, 1990), qui relie la personne à son environnement humain, social et naturel, les savoirs à leurs conditions de production, à leurs aptitudes à générer des processus de problématisation et à tisser des liens entre les phénomènes du monde, et finalement à donner du sens à une expérience de vie.

Opter pour une transposition de pratiques de recherche humbles et pragmatiques, plutôt qu'une science dont l'excellence serait mesurée exclusivement à l'aulne de palmarès internationaux.

Nous avons mis en évidence trois balises qui nous semblent incontournables pour concevoir l'enseignement de l'agroécologie, il s'agit d'organiser les conditions pédagogiques et didactiques de : l'étude des enjeux associés aux pratiques de production, de transformation et de consommation des produits de l'agriculture au regard des défis environnementaux et sociaux; l'explicitation de la nature de la science en agroécologie dans ses dimensions épistémique, socio-institutionnelle et son environnement culturel et social; la mise en démarche d'enquête des élèves à propos d'une question agroécologique locale. Ces trois balises nous apparaissent cohérentes avec les résultats de nos entretiens qui montrent la transdisciplinarité que les chercheurs mettent en œuvre pour appréhender la complexité des objets de savoirs, l'importance des études de cas pensées sur un mode participatif à la fois dans la problématisation et les propositions d'actions, le travail sur la cohérence à trouver entre valeurs personnelles et scientifiques. Ces trois balises nous semblent de nature à contribuer d'un point de vue didactique au renouvellement épistémologique que Coutellec (2015) nomme " sciences impliquées ». De plus, ces trois balises nous semblent participer à la construction chez les élèves d'un questionnement sur le rapport qu'ils entretiennent au vivant (un vivant cultivé, domestiqué ou sauvage) et sur la manière dont ils envisagent les relations sciences-société (une science toute à la fois «compliquée », " expliquée » et “ impliquée »). 
Nous souhaitons continuer ce travail de transposition, en particulier du point de vue de la recontextualisation, c'est-à-dire en positionnant ces éléments de la nature de la science dans un contexte pédagogique. Pour cela, plusieurs principes fondent la démarche que nous sommes en train de mettre en place : c'est au travers d'une collaboration avec des enseignants que la recontextualisation prendra son sens, celle-ci s'appuiera en partie sur des extraits de nos entretiens filmés, et il s'agira de questionner également la dépersonnalisation du savoir, considérée comme une caractéristique majeure de la transposition didactique externe (Chevallard, 1985).

Ainsi, le chercheur en didactique se situe à différents niveaux de la transposition didactique externe, en mettant en relation le monde de la recherche et le monde de l'enseignement. C'est peut-être ainsi qu'il faut entendre le changement de posture épistémologique que souhaitent El Hage \& Ouvrier-Buffet (2018), les recherches collaboratives qui se mettent en place entre enseignants et chercheurs en didactique d'un côté de la chaîne de transposition pourraient également se décliner entre chercheurs en didactique et chercheurs sur les savoirs à enseigner de l'autre côté de la chaîne de transposition. Cette position d'interface du didacticien renforcerait sans doute sa fonction sociale, et permettrait peut-être de légitimer davantage les pédagogies de l'enquête.

\section{Bibliographie}

Abd-El-Khalick, F. (2012). Examining the sources for our understandings about science: Enduring conflations and critical issues in research on nature of science in science education. International Journal of Science Education, 34(3), 353-374. https://doi.org/10.1080/09500693.2011.629013

Albe, V., et Orange, C. (2010). Sciences des scientifiques et sciences scolaires. Revue de Didactique des Sciences et Techniques, (2), 19-26.

Allchin, D. (2013). Teaching the nature of science: Perspectives and resources. St. Paul, MN: SHiPS Education Press.

Allchin, D. (2017). Beyond the Consensus View : Whole Science. Canadian Journal of Science, $\begin{array}{llll}\text { Mathematics and Technology Education, 17(1), } & \text { 18-26. }\end{array}$ https://doi.org/10.1080/14926156.2016.1271921

Alsop, S., et Gardner, S. (2017). Opening the Black Box of NOS: Or Knowing How to Go On With Science Education, Wittgenstein, and STS in a Precarious World. Canadian Journal of Science, Mathematics and Technology Education, 17(1), 27-36. https://doi.org/10.1080/14926156.2016.1271924

Astolfi, J.-P., et Develay, M. (1989). La didactique des sciences. Paris : Presses Universitaires de France.

Balacheff, N. (2003). CK\$ Modèle de connaissances pour le calcul de situations didactiques. Dans A. Mercier et C. Margolinas (Dir.), Balises pour la didactique des mathématiques. Cours de la Xlle École d'été de didactique de mathématiques (p. 1-32). Grenoble : La pensée sauvage.

Bardin, L. (2013). L'analyse de contenu. Paris: Presses Universitaires de France.

Bencze, L., Pouliot, C., Pedretti, E., Simonneaux, L., Simonneaux, J., et Zeidler, D. (2020). SAQ, SSI and STSE education: Defending and extending "science-in-context". Cultural Studies of Science Education. https://doi.org/10.1007/s11422-019-09962-7

Calmettes, B. (2012). Modélisation pragmatiste de l'action didactique de l'enseignant. Le cas de la démarche d'investigation en collège. Habilitation à diriger des recherches en Sciences de l'Éducation. Université de Toulouse.

Cancian, N., et Simonneaux, J. (2019). Les objets intermédiaires agroécologiques à enquêter. Dans J. Simonneaux (dir.), La démarche d'enquête. Une contribution à la didactique des questions socialement vives (p. 186-206). Dijon : Educagri éditions.

Chateauraynaud, F. (2011). Argumenter dans un champ de forces. Essai de balistique sociologique. Paris : éditions Petra.

Chevallard, Y. (1985). La transposition didactique. Du savoir savant au savoir enseigné. Grenoble : La pensée sauvage. 
Claverie, B. (2010). Pluri-, inter-, transdisciplinarité : Ou le réel décomposé en réseaux de savoir. Projectics / Proyéctica / Projectique, 4(1), 5-27. https://doi.org/10.3917/proj.004.0005

Coutellec, L. (2015). La science au pluriel: Essai d'épistémologie pour des sciences impliquées. Versailles : Quae éditions.

Direction générale de l'enseignement et de la recherche. (2014). Plan d'action Enseigner à produire autrement. Paris: Ministère de l'Agriculture et de l'alimentation. Repéré à https://chlorofil.fr/eapa/plan

Direction générale de l'enseignement et de la recherche. (2020). Plan d'action Enseigner à produire autrement 2. Paris : Ministère de l'Agriculture et de l'alimentation. Repéré à https://chlorofil.fr/eapa

Duru, M., Therond, O., Martin, G., Martin-Clouaire, R., Magne, M.-A., Justes, E., ... Sarthou, J. P. (2015). How to implement biodiversity-based agriculture to enhance ecosystem services: A review. Agronomy for Sustainable Development, 35(4), 1259-1281. https://doi.org/10.1007/s13593-015-0306-1

El Hage, S., et Ouvrier-Buffet, C. (2018). Les démarches de chercheurs en physique et en mathématiques. Enjeux didactiques d'une nouvelle approche épistémologique. , 34, 6282. Recherches en éducation, (34), 62-82.

El Hage, S., et Plé, E. (2016). Démarches d'investigation en sciences: Des références épistémologiques questionnées au regard de la science qui se pratique. Dans Actes des ges rencontres scientifiques de l'Association de Recherche en Didactiques, Sciences et Technologies (ARDIST). Lens. Repéré à https://ardist2016lens.sciencesconf.org/82701/document

Erduran, S., et Dagher, Z. (2014). Reconceptualizing the Nature of Science for Science Education. Dordrecht, The Netherlands: Springer.

Fabre, M. (2009). Pédagogie et philosophie du problème. Paris : Vrin.

Fabre, M. (2011). Éduquer pour un monde problématique. La carte et la boussole. Paris : Presses Universitaires de France.

Fabre, M. (2017). Problématisation-Problèmes flous. Dans A. Barthes, J.-M. Lange, et N. Tutiaux-Guillon (Dir.). Dictionnaire critique des enjeux et concepts des éducations à... (p. 523-529). Paris : L'Harmattan.

Francis, C., Lieblein, G., Gliessman, S., Breland, T. A., Creamer, N., Harwood, R., ... Poincelot, R. (2003). Agroecology: The Ecology of Food Systems. Journal of Sustainable Agriculture, 22(3), 99-118. https://doi.org/10.1300/J064v22n03_10

Frère, N. (2017). Les trajectoires de positionnements sur les différentes approches agroécologiques d'apprenants en formation agricole incluant un module d'agroécologie Thèse de doctorat en Sciences de l'Éducation. Université de Toulouse.

Gardes, M.-L. (2013). Étude de processus de recherche de chercheurs, élèves et étudiants, engagés dans la recherche d'un problème non résolu en théorie des nombres Thèse de doctorat en Sciences de l'Éducation. Université Claude Bernard - Lyon 1.

Geels, F. W., et Schot, J. (2007). Typology of sociotechnical transition pathways. Research policy, 36(3), 399-417. https://doi.org/10.1016/j.respol.2007.01.003

Gendron, C. (2014). Penser l'acceptabilité sociale: Au-delà de l'intérêt, les valeurs. Communiquer, (11), 117-129.

Gliessmann, S. R. (1998). Agroecology: Ecological Processes in Sustainable Agriculture. Chelsea, Michigan: Ann Arbor Press.

Hodson, D. (2011). Looking to the Future: Building a Curriculum for Social Activism. Rotterdam: Sense Publishers.

Hodson, D., et Wong, S. L. (2017). Going Beyond the Consensus View : Broadening and Enriching the Scope of NOS-Oriented Curricula. Canadian Journal of Science, Mathematics and Technology Education, 17(1), 3-17.

Irzik, G., et Nola, R. (2014). New Directions for Nature of Science Research. Dans M. R. Matthews (Dir.), International Handbook of Research in History, Philosophy and Science Teaching (p. 999-1021). Dordrecht: Springer Netherlands. https://doi.org/10.1007/97894-007-7654-8_30 
Joshua, S. (1996). Le concept de transposition didactique n'est-il propre qu'aux mathématiques? Dans C. Raisky et M. Caillot (Dir), Au-delà des didactiques, le didactique. Débats autour de concepts fédérateurs (p. 61-73). Bruxelles, Belgique : De Boeck Supérieur.

Kind, P. E. R., \& Osborne, J. (2016). Styles of scientific reasoning: A cultural rationale for science education? Science Education, 101(1), 8-31.

Lange, J.-M., et Victor, P. (2006). Didactique curriculaire et «éducation à... La santé, l'environnement et au développement durable »: Quelles questions, quels repères ? Aster, 28, 85-100.

Latour, B. (1989). La science en action. Paris : La découverte.

Lederman, N. G. (2007). Nature of Science: Past, Present, and Future. Dans S. K. Abell \& N.G. Lederman (Dir.), Handbook of research on science education (p. 831-879). Mahwah, New Jersey: LEA Editions.

Matthews, M. (2012). Changing the focus: From nature of science (NOS) to features of science (FOS). Dans M. S. Khine (Dir.), Advances in nature of science research (p. 3-26). Dordrecht, The Netherlands: Springer.

McComas, W. F., Clough, M. P., et Almazroa, H. (1998). The Role and Character of the Nature of Science in Science Education. Dans W. F. McComas (Dir.), The Nature of Science in Science Education: Rationales and Strategies (p. 3-39). Boston: Kluwer Academic Publishers.

Métral, J. F., Olry, P., Prévost, P., Cancian, N., Frère, N., et Simonneaux, L. (2016). Ruptures ou ajustements provoqués entre pratiques agricoles et enseignement de ces pratiques. Formation emploi, 135, 53-74.

Paun, E. (2006). La transposition didactique : Un processus de construction du savoir scolaire. Carrefours de l'éducation, 22, 3-13.

Pommier, M., Foucaud-Scheunemann, C., et Morel-Deville, F. (2010). De la recherche à l'enseignement: Modalités du partage des savoirs dans le domaine des sciences de la vie et de la Terre. Recherches en Didactique des Sciences et des Techniques, 2, 127-156.

Prévost, P., Métral, J.-F., Simonneaux, L., Cancian, N., Chrétien, F., David, M., et Olry, P. (2018). Approche plurididactique pour l'élaboration curriculaire dans l'enseignement des sciences techniques en formation professionnelle : Propositions à partir de l'exemple de l'agronomie. Éducation \& didactique, 12(2), 53-71.

Reboud, X., et Hainzelin, É. (2017). L'agroécologie, une discipline aux confins de la science et du politique. Natures Sciences Sociétés, Supplément(Supp. 4), 64-71. https://doi.org/10.1051/nss/2017036

Rosset, P. M., et Martinez-Torres, M. E. (2012). Rural Social Movements and agroecology Context, Theory, and Process. Ecology and Society, 17(3), 1-12.

Simonneaux, L, Simonneaux, J., Hervé, N., Molinatti, G., Nedelec, L., Cancian, N., et Lipp, A. (2017). Menons l'enquête sur des questions d'EDD dans la perspective des QSV. Formation et pratiques d'enseignement en questions, 22, 143-160.

Simonneaux, L. (2017). Au-delà de la polémique, compléter l'approche macro consensuelle de la NOS avec l'approche micro de la recherche en train de se faire. Canadian Journal of Science, Mathematics and Technology Education, 17(1), 58-65. https://doi.org/10.1080/14926156.2016.1271922

Sjöström, J., Frerichs, N., Zuin, V. G., \& Eilks, I. (2017). Use of the concept of Bildung in the international science education literature, its potential, and implications for teaching and learning. Studies in Science Education, 53(2), 165-192. https://doi.org/10.1080/03057267.2017.1384649

Stassart, P. M., Baret, P., Grégoire, J.-C., Hance, T., Mormont, M., Reheul, D., ... Visser, M. (2012). L'agroécologie: Trajectoire et potentiel. Pour une transition vers des systèmes alimentaires durables. Dans D. Van Dam, M. Streith, J. Nizet, et P. M. Stassart (Dir.), Agroécologie, entre pratiques et sciences sociales (p. 25-51). Dijon : Éducagri éditions.

Tiberghien, A. (2011). Conception et analyse de ressources d'enseignement: Le cas des démarches d'investigation. Dans M. Grangeat (Dir.), Les démarches d'investigation dans 
l'enseignement scientifique. Pratiques de classe, travail collectif enseignant, acquisitions des élèves (p. 185-212). Lyon : ENS Éditions.

Tytler, R. (2007). Re-imagining Science Education: Engaging students in science for Australia's future (Rapport No. 51). Camberwell: Australian Council for Education Research.

Veillard, L., Tiberghien, A., et Vince, J. (2011). Analyse d'une activité de conception collaborative de ressources pour l'enseignement de la physique et la formation des professeurs. Le rôle de théories ou outils spécifiques. Activités, 08(8-2), 202-227. https://doi.org/10.4000/activites.2627

Vinck, D. (2007). Présentation. Cet obscur objet de connaissances. Revue d'anthropologie des connaissances, 1, 1, 5-10. https://doi.org/10.3917/rac.001.0005

Wezel, A., Bellon, S., Doré, T., Francis, C., Vallod, D., et David, C. (2009). Agroecology as a science, a movement and a practice. A review. Agronomy for Sustainable Development, 29(4), 503-515. https://doi.org/10.1051/agro/2009004

Wong, S. L., Hodson, D., Kwan, J., et Yung, B. H. W. (2008). Turning Crisis into Opportunity: Enhancing student- teachers' understanding of nature of science and scientific inquiry through a case study of the scientific research in severe acute respiratory syndrome. International Journal of Science Education, 30(11), 1417-1439. https://doi.org/10.1080/09500690701528808

Wong, S. L., Kwan, J., Hodson, D., et Yung, B. H. W. (2009). Turning Crisis into Opportunity: Nature of Science and Scientific Inquiry as Illustrated in the Scientific Research on Severe Acute Respiratory Syndrome. Science \& Education, 18(1), 95-118. https://doi.org/10.1007/s11191-007-9123-5 


\section{ANNEXE 1 : guide d'entretien}

$\left.1^{\circ}\right)$ Quelle est votre activité du moment?

$2^{\circ}$ ) Vous êtes chercheur, vous cherchez quoi ?

Objets, méthodes

$\left.3^{\circ}\right)$ Comment vous décririez votre métier aujourd'hui ?

Activités solitaires - collectives

$\left.4^{\circ}\right)$ Quelles sont les grandes étapes structurantes de votre parcours de chercheur?

$\left.5^{\circ}\right)$ Si on s'intéresse au contexte de l'activité scientifique, comment votre métier a évolué ? Politique scientifique, Financement, Globalisation des projets, lien avec l'industrie ou start-up, transdisciplinarité, construction des contrats, animation scientifique

$\left.6^{\circ}\right)$ Qu'est-ce qu'on demande aujourd'hui aux chercheurs ?

Dimension institutionnelle (communication, production de savoirs, financement) - Dimension sociétale (responsabilité du chercheur, co-construction des objets/résutats de recherche, enjeux de société, engagement social)

$7^{\circ}$ ) Vous en tant que producteur de savoirs scientifiques en lien avec l'agriculture, qu'est-ce que vous pensez de vos rapports avec les acteurs du monde agricole?

Votre rôle social, ce que vous pensez des enjeux du vivant (écologie, OGM, artificialisation des sols, controverses)

$\left.8^{\circ}\right)$ Quels sont les savoirs qui vous semblent les plus importants dans votre activité de chercheur? Dans les enjeux de société actuels?

Engagement, différents acteurs, changements de modèle agricole, projection dans le futur

$\left.9^{\circ}\right)$ Qu'est-ce qu'il serait important à vos yeux de transmettre aux jeunes, aux élèves ?

Savoirs, pratiques, valeurs 